\title{
Advanced Cyclic Coding Technique for Long-Range Raman DTS Systems with Meter-Scale Spatial Resolution over Standard SMF
}

\author{
Marcelo A. Soto, Tiziano Nannipieri, Alessandro \\ Signorini, Gabriele Bolognini*, and Fabrizio Di Pasquale \\ Scuola Superiore Sant'Anna \\ Via G. Moruzzi 1, 56124 Pisa, Italy \\ *g.bolognini@sssup.it
}

\begin{abstract}
A long-range fast-measurement Raman-based distributed temperature sensor (RDTS) with a meter spatial resolution exploiting a quasi-periodic pulse-coding technique is proposed in this paper. We experimentally demonstrate the feasibility of temperature sensing over $\mathbf{2 6}$ $\mathrm{km}$ standard single-mode fiber with $1 \mathrm{~m}$ spatial resolution obtaining $3{ }^{\circ} \mathrm{C}$ of temperature resolution within $30 \mathrm{~s}$ of measurement time. Experimental results employing 1023bits codewords are also reported, showing a notable range enhancement and enabling distributed sensing over $58 \mathrm{~km}$ distance with $2 \mathrm{~m}$ spatial resolution and $4{ }^{\circ} \mathrm{C}$ of temperature resolution within 5 min measurement time.
\end{abstract}

\section{INTRODUCTION}

Distributed temperature sensors (DTS) constitute an effective solution for many industrial applications, such as power cables monitoring or leakage detection in oil\&gas pipelines. Conventional Raman-DTS (RDTS) are mostly based on optical time-domain reflectometry (OTDR), where the temperature estimation over the whole fiber is provided by injecting optical pulses into the sensing fiber and detecting the backscattered Stokes (or Rayleigh) and anti-Stokes signals. However, the extremely low power of the Raman backscattered signals requires the use of high sensitivity photodiodes and the acquisition of many traces in order to increase the signal-to-noise ratio (SNR) of the measurements. The use of multi-mode fibers (MMFs) as a sensing medium allows for the partial mitigation of the noise impact thanks to the higher backscattering and higher nonlinear threshold that characterize MMFs compared to single-mode fibers (SMFs), but the large modal dispersion degrades the performance in terms of spatial resolution at long distance ranges. However, in many applications spanning long sensing ranges, good spatial and temperature resolutions combined with short acquisition times are required. The use of optical pulse coding techniques applied to RDTS systems has been demonstrated to provide a significant SNR improvement; however, this method does not necessarily allow for high spatial/temperature resolutions and short measurement time. This is because, the method has been basically proposed for RDTS system operating over MMFs [1] or over SMFs [2] using externally modulated semiconductor lasers. While MMFs allow for higher temperature resolution values and longer distances with

\author{
Andrea Lazzeri, Federico Baronti, and Roberto Roncella \\ Department of Information Engineering \\ University of Pisa \\ Via G. Caruso 16, 56126 Pisa, Italy
}

reduced spatial resolution values, the low backscattering levels as well as the relatively low power (a few hundred $\mathrm{mW}$ ) of semiconductor lasers partially cancel out the benefits brought by coding in terms of SNR enhancement when using SMFs. In particular for RDTS systems operating on standard SMFs with pulse coding, the maximum usable peak power level before onset of nonlinearities is approximately $\sim 3-4 \mathrm{~W}$, which is far above the maximum power of semiconductor lasers. Then, the use of new coding schemes applied to SMF-based RDTS systems exploiting high-power pulsed lasers (such us Qswitched and rare-earth doped fiber lasers) would have a significant impact on RDTS applications.

In this work we exploit the quasi periodic property of Simplex cyclic pulse coding technique for the experimental investigation of the benefits in term of sensing distance capabilities and measurement time with respect to the conventional single-pulse technique using SMFs. We experimentally verify the sensing capabilities of an RDTS system using $26 \mathrm{~km}$ of standard SMF and 71-bit codeword length achieving $3{ }^{\circ} \mathrm{C}$ of temperature resolution within $30 \mathrm{~s}$ measurement time and with $1 \mathrm{~m}$ spatial resolution. We also experimentally investigate on the performance in terms of sensing range extension using a 1023-bits Simplex codewordlength, achieving, for the first time to our knowledge, an RDTS over a sensing distance of $58 \mathrm{~km}$ with $2 \mathrm{~m}$ spatial resolution and $4{ }^{\circ} \mathrm{C}$ of temperature resolution within $5 \mathrm{~min}$ measurement time.

\section{THEORY}

In RDTS systems an optical pulse is sent down along an optical fiber and the spontaneous Raman scattering (SpRS) is measured using OTDR techniques. Although the intensity of the anti-Stokes SpRS component exhibits a strong dependence on the fiber temperature (while the Stokes SpRS component is only slightly temperature dependent), the OTDR traces of the anti-Stokes component only is not commonly used for temperature sensing, since traces also depend on fiber losses, whose variations might be easily interpreted as a temperature change, inducing errors in the measurement. In order to overcome this problem, the antiStokes intensity trace has to be normalized by a temperatureindependent trace, such as the Stokes SpRS or Rayleigh component [3-4], so that local fiber loss effects are cancelled 
out. Unfortunately, the intensity of the SpRS is very low (of the order of 60-70 dB below the peak power used for fiber interrogation); therefore, the backscattered optical power impacting the receiver is very low, limiting significantly the temperature resolution of the measurements. Actually, the ultimate parameter determining the temperature resolution is the electrical SNR. Some techniques based on optical pulse coding have been proposed to enhance the SNR of RDTS systems [2], as well as of Brillouin-based distributed optical fiber sensors [5]. In those cases, to achieve 1 meter spatial resolution, a CW light is intensity modulated by sequences of $10 \mathrm{~ns}$ pulses, i.e. with a modulation frequency of $100 \mathrm{MHz}$. Unfortunately this frequency is incompatible with the laser technology used for high-performance RDTS systems, which is characterized by high peak power (several tens of Watt), low repetition rates (a few hundreds $\mathrm{kHz}$ ) and very low duty cycles (typically $0.1 \%$ ). To overcome this limitation, recently a new coding technique based on cyclic Simplex codes has been theoretically proposed and recently experimentally validated in [1]. The method consists in launching optical pulses into the fiber at a low repetition rate, 'filling' the whole sensing fiber with a large number of bits.

The main advantage of cyclic codes for RDTS applications using high-power lasers, with respect to other coding schemes, is their compatibility with the use of low-repetition rate lasers, exhibiting very low duty cycle values. They are based on a quasi-periodic intensity modulation pattern of the laser light, requiring only an additional external modulator to be used as a fast chopper when direct pulsed laser modulation cannot be implemented. In the case cyclic Simplex codes pattern is employed, an SNR enhancement equal to $(L+1) /(2 \sqrt{ } L)$ is expected (where $L$ is the code length) [1]. In this work, the benefits provided by cyclic coding techniques have been further investigated. Firstly, we show the sensing capabilities of an RDTS operating over $26 \mathrm{~km}$-long SMF, using $1 \mathrm{~m}$ spatial resolution. Then, by using a 1023 bit Simplex codeword, an extended-range coded RDTS is implemented; achieving what we believe is the first demonstration of Raman-based distributed temperature sensing over $58 \mathrm{~km}$-long SMF using $2 \mathrm{~m}$ spatial resolution. This coding method can potentially allow for RDTS systems with sub-meter spatial resolution using conventional timedomain techniques.

\section{CODED-RDTS WITH 1 METER SPATIAL RESOLUTION} ALONG A 26 KM SINGLE-MODE FIBER

\section{A. Experimental setup}

The setup used in the experiment is shown schematically in Fig. 1. The light source is a high-power rare-earth doped fiber laser (50 W maximum peak power) centered at $1550 \mathrm{~nm}$, with $10 \mathrm{~ns}$ pulsewidth (allowing for an attainable spatial resolution of $1 \mathrm{~m}$ ) and a maximum repetition rate of $\sim 250$ $300 \mathrm{kHz}$. To avoid nonlinear effects, the peak power injected into the sensing fiber has been adjusted by using a variable optical attenuator (VOA). An acousto-optic modulator (AOM), which is triggered by an FPGA-based generator, is used to generate the cyclic Simplex codeword to be launched

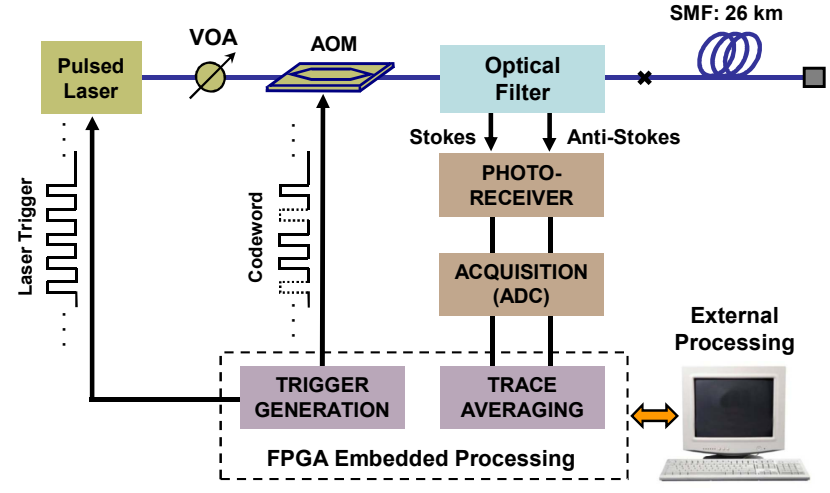

Figure 1. Experimental setup for an RDTS system over $26 \mathrm{~km}$ singlemode fiber, using 71 bit Simplex coding.

into the fiber, stopping laser pulses (' 0 ' bits) or letting them through (' 1 ' bits). The burst of pulses is sent into a $26 \mathrm{~km}$ standard SMF by using a suitable optical filter, also allowing us to separate the backscattered Raman Stokes and anti-Stokes components into two parallel branches at the receiver. Each receiver-branch is composed of a high-sensitivity avalanche photodiode (APD), a trans-impedance and gain amplifier stages, and an FPGA-controlled analog-to-digital converter (ADC) which is connected to a computer for data processing.

\section{B. Results}

To estimate the SNR enhancement provided by the cyclic codes, measurements with coding are compared to the ones obtained by the conventional technique, using the same peak power and same acquisition time. Allocating some power margin before the onset of nonlinearities (occurring at $\sim 37 \mathrm{dBm}$ power), we employed pulse peak power of $35 \mathrm{dBm}$. Considering that the used optical fiber is $26 \mathrm{~km}$-long, the repetition rate of the laser has been set to $\sim 230 \mathrm{kHz}$, allowing us to allocate up to 71 bits inside the fiber. Fig. 2 shows the acquired coded anti-Stokes trace, where we can observe the repetition period of the bits every $435 \mathrm{~m}$, according to the laser repetition rate. After a linear decoding process, the single-pulse fiber response for the anti-Stokes and Stokes components is recovered with an enhanced SNR with respect to the conventional single-pulse technique. Actually, in Fig. 3 the normalized anti-Stokes trace measured with the standard scheme is compared with the one obtained after decoding the Simplex-coded traces, both measured with the same number of acquisitions (100k time-averaged traces) and the same measurement time (about $30 \mathrm{~s}$ ). The experimentally achieved

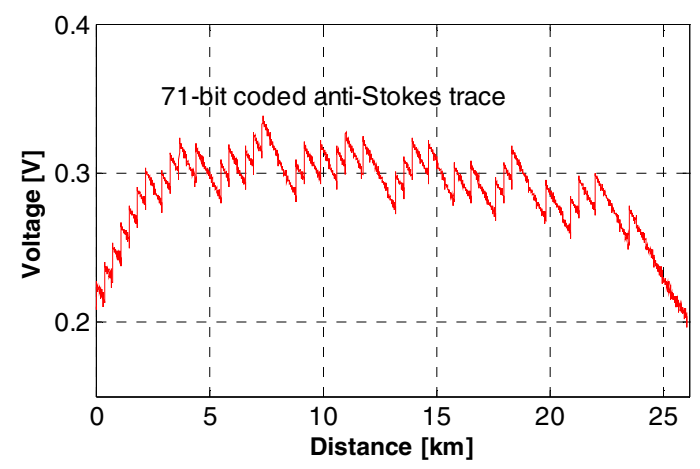

Figure 2. Coded anti-Stokes trace, with 71 bit Simplex cyclic codes. 


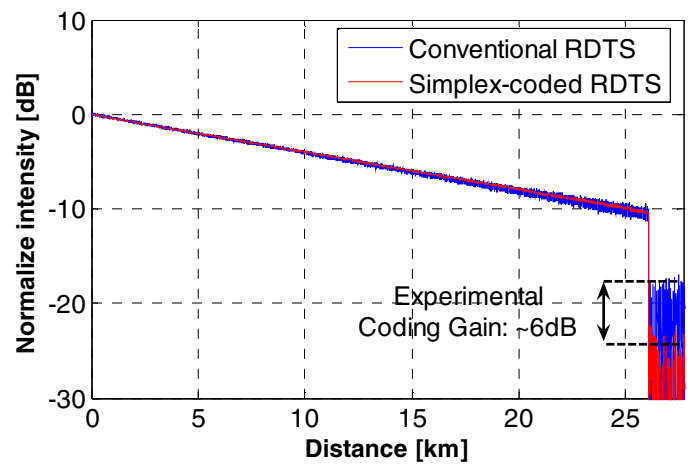

Figure 3. Anti-Stokes traces obtained with both standard single-pulse technique and cyclic Simplex coding (trace after decoding).

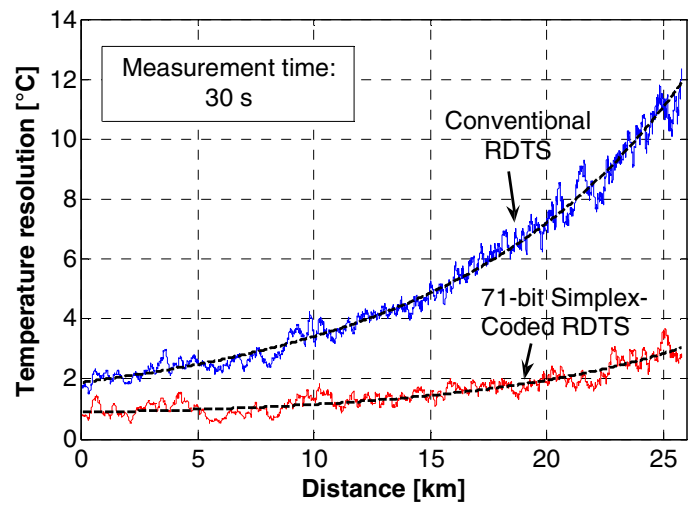

Figure 4. Temperature resolution as a function of the distance, for both single-pulse and cyclic-Simplex coded RDTS.

SNR enhancement was $6.0 \mathrm{~dB}$, which is in good agreement with the theoretical coding gain $(6.3 \mathrm{~dB})$.

Note that in the conventional case, the use of SMFs leads to a relatively low electrical SNR, an issue that strongly impacts on the final temperature resolution of the RDTS, as reported in Fig. 4. We can observe that the use of SMFs in conventional RDTS systems provides a poor temperature resolution, which in our case is equal to $12{ }^{\circ} \mathrm{C}$ at $26 \mathrm{~km}$ distance; the use of the distributed cyclic optical coding improves the resolution down to $3{ }^{\circ} \mathrm{C}$ at the same distance. If we compare with the conventional RDTS, where a $3{ }^{\circ} \mathrm{C}$ temperature resolution is achieved at $\sim 8 \mathrm{~km}$ distance, we can conclude that the proposed technique offers a sensing range enhancement of about $18 \mathrm{~km}$ (when 71 bit are used), a value which is in agreement with the coding gain and the two-way SMF losses $(\sim 0.2 \mathrm{~dB} / \mathrm{km}$ at $1550 \mathrm{~nm})$. It is important to point out that the use of SMFs and distributed cyclic coding allows for temperature resolution values of the same order as what is reported in the literature using MMFs and conventional RDTS [4]. This is a very important feature, since the cyclic coding technique enables already-installed SMFs to be used for longrange RDTS, without the need of installing new cables containing MMFs, and also providing better spatial resolution. To estimate the real spatial resolution achieved in our experiment, the last few meters of fiber (near $26 \mathrm{~km}$ distance) have been placed inside a temperature-controlled chamber which has been set to $55^{\circ} \mathrm{C}$, while the rest of the fiber is kept at $25^{\circ} \mathrm{C}$ (room temperature). Fig. 5 shows the temperature variation of $30^{\circ} \mathrm{C}$, where a spatial resolution of $\sim 1.0 \mathrm{~m}$ can be

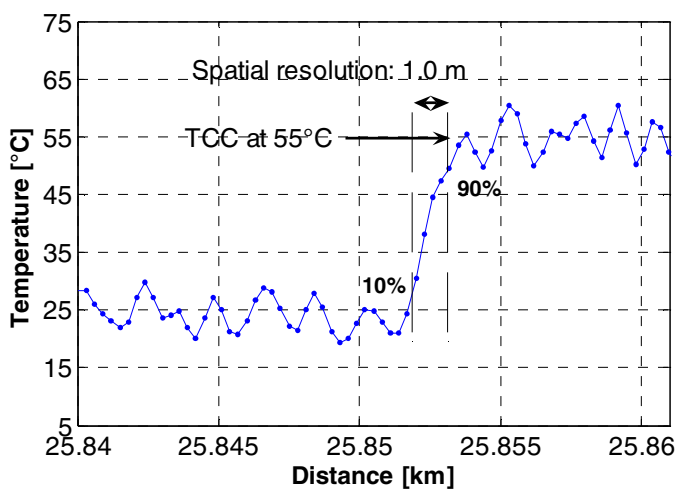

Figure 5. Temperature profile as a function of the distance, near the far fiber-end ( $\sim 26 \mathrm{~km}$ distance), when TCC is set to $55^{\circ} \mathrm{C}$.

observed (calculated as the distance corresponding to a $10 \%$ $90 \%$ of the temperature change).

Comparing the amount of pulse dispersion for $10 \mathrm{~ns}$ pulses after propagation along SMFs and MMFs of similar length (26 km for our case), in SMF the pulse experiences a minimum temporal spread due to chromatic dispersion only $(\sim 15 \mathrm{ps} / \mathrm{nm} \cdot \mathrm{km}$ around $1550 \mathrm{~nm})$, resulting in an expected spread smaller than $0.04 \mathrm{~ns}$ at the far fiber-end (laser linewidth $\sim 0.1 \mathrm{~nm}$ ). On the other hand, when using MMFs the higher modal dispersion (up to several $\mathrm{ns} / \mathrm{km}$ for graded-index fibers [6]) induces a significantly higher expected pulse broadening ( $\sim 26 \mathrm{~ns}$ at fiber end), exhibiting also a great variability depending on mode coupling conditions into MMF. Considering that the backscattered light is also affected by similar spread, doubling the impact of dispersion, the spatial resolution of RDTS with MMFs is degraded to values up to $5 \mathrm{~m}$ at $26 \mathrm{~km}$, significantly worse than with SMFs, which allow for an estimated resolution smaller than $1.08 \mathrm{~m}$ (as confirmed by the resolution of $\sim 1.0 \mathrm{~m}$ reported in Fig. 4 ).

\section{CODED-RDTS WITH 2 METER SPATIAL RESOLUTION ALONG A 58 KM SINGLE-MODE FIBER}

\section{A. Experimental setup}

Considering that the repetition rate of the laser used in Fig. 1 can be increased only up to $\sim 250-300 \mathrm{kHz}$, the maximum number of bits that is possible to allocate in the fiber is limited, constraining the SNR enhancement that the cyclic-coding technique can provide to the RDTS system. Hence, in order to further extend the sensing distance of coded-RDTSs, the generation of the pulse sequences

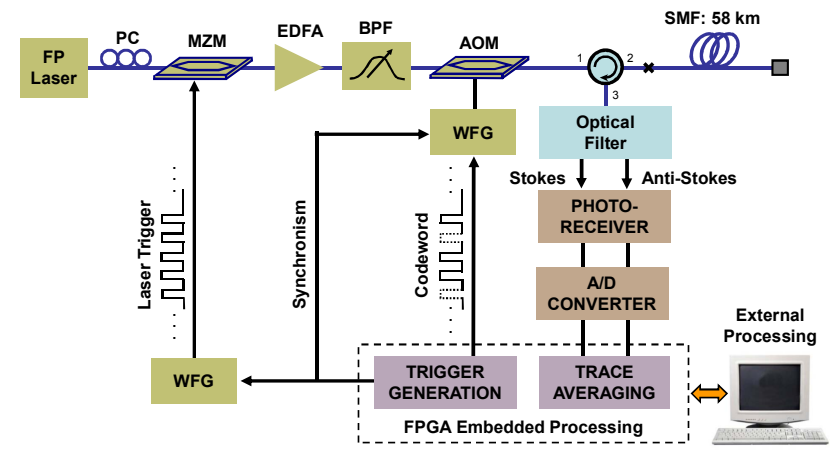

Figure 6. Experimental setup for an RDTS system over a $58 \mathrm{~km}$ singlemode fiber, using 1023 bit Simplex coding. 
(codeword) has to be implemented using another alternative mechanism. In particular, we have used the experimental setup shown in Fig. 6. The optical source is a Fabry-Perot laser with $\sim 10 \mathrm{~nm}$ linewidth, which is externally-modulated by a MachZehnder modulator (MZM). In this way, pulses with $20 \mathrm{~ns}$ length and a repetition period of $580 \mathrm{~ns}$ have been generated and then amplified by an erbium-doped fiber amplifier (EDFA). Considering the pulse repetition rate of $\sim 1.72 \mathrm{MHz}$, no transient effects in the EDFA have been observed, and therefore, every pulse has the same peak power at the output of the EDFA. Then, an AOM is used to generate the codeword to be launched into the fiber. Note that no VOA is used in this case because the pulse peak power can be easily controlled by adjusting the pump power in the EDFA. The rest of the setup is similar to the previously used (reported in Fig. 1). However, the length of the sensing fiber has been considerably increased up to $58 \mathrm{~km}$, allowing us to use a pulse sequence of $1023 \mathrm{bit}$.

\section{B. Results}

In order to avoid nonlinear effects, the peak pulse power that is launched into the fiber has been adjust to $\sim 34 \mathrm{dBm}$ (note that the SRS threshold is expected to be reduced with respect to the case of $10 \mathrm{~ns}$ pulses [7]). It is important to point out that Raman-based temperature sensing along $58 \mathrm{~km}$ single-mode fiber is a challenging task. Actually, long-range RDTS are typically based on the use of MMFs; the usual method to improve SNR is increasing the number of timeaveraged traces, inducing a critical increase in the measurement time. The use of pulse coding allows for overcoming this problem, offering a significant reduction in the acquisition time. In our experiment we used 1023 bits, offering a SNR enhancement of $\sim 12 \mathrm{~dB}$, which can be employed to reduce the total measurement time by more than $90 \%$. To verify the ultra-long-range sensing capabilities, the temperature of the last meters of fiber (near $58 \mathrm{~km}$ distance) was increased from room temperature $\left(28^{\circ} \mathrm{C}\right)$ up to $55^{\circ} \mathrm{C}$ using a TCC. Figure 8 reports the first results of temperature measurement along $58 \mathrm{~km}$ distance using an RDTS system with $2 \mathrm{~m}$ spatial resolution and $500 \mathrm{k}$ time-averaged traces, corresponding to less than $5 \mathrm{~min}$ measurement time. The estimated temperature resolution has been found to be $\sim 4{ }^{\circ} \mathrm{C}$ at $58 \mathrm{~km}$ distance. If the measurement time is increased to $10 \mathrm{~min}$, the temperature resolution can be improved down to $\sim 2.9^{\circ} \mathrm{C}$. Similar temperature resolution values could be obtained by a conventional single-pulse RDTS only by using very long measurement times, such as $\sim 80 \mathrm{~min}$ and $\sim 160 \mathrm{~min}$ for $\sim 4{ }^{\circ} \mathrm{C}$ and $\sim 2.9^{\circ} \mathrm{C}$ resolution respectively.

\section{CONCLUSION}

In conclusion, we have experimentally demonstrated the benefits of advanced coding techniques based on cyclic coding to implement long-range RDTS systems using pulsed laser technology. This method enables the use of SMFs for longrange temperature sensing, overcoming the spatial resolution degradation typically affecting long-range RDTS systems based on MMFs. Simplex cyclic coding allowed us to implement, for the first time to our knowledge, fast Ramanbased temperature sensing with $1 \mathrm{~m}$ spatial resolution along $26 \mathrm{~km}$ of SMF, with a temperature resolution of $3{ }^{\circ} \mathrm{C}$ and

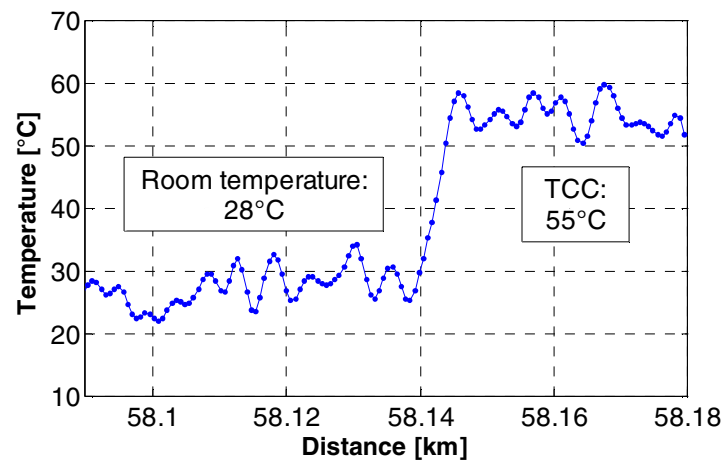

Figure 7. Temperature measurement along $58 \mathrm{~km}$ sensing distance when a few meters of fiber are heated up to $55^{\circ} \mathrm{C}$ (for clarity only the last meters of fiber are shown)

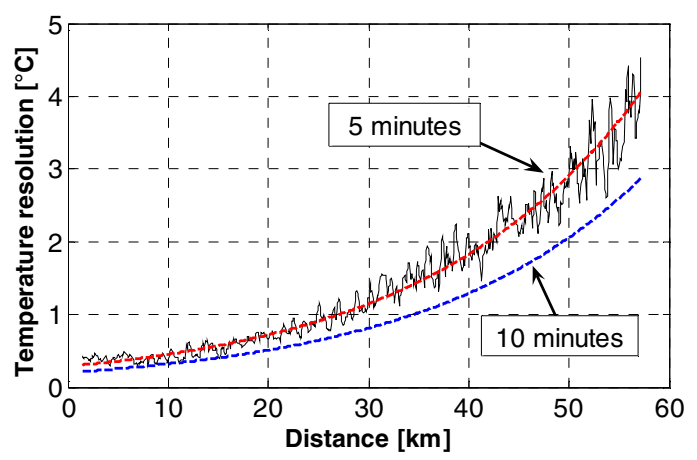

Figure 8. Temperature resolution versus distance, when 1023 bit cyclicSmplex coding is used.

$\sim 30$ s measurement time. this result, achieved using standard high-power pulsed lasers, represents a sensing distance enhancement of $18 \mathrm{~km}$ with respect to standard RDTS. Further range enhancement has been also achieved using a larger number of pulses (1023 bit) achieving temperature sensing along $58 \mathrm{~km}$ SMF distance with $4{ }^{\circ} \mathrm{C}$ resolution, $5 \mathrm{~min}$ measurement time and $2 \mathrm{~m}$ spatial resolution capabilities.

\section{REFERENCES}

[1] M. A. Soto et al., "Raman-based distributed temperature sensor with $1 \mathrm{~m}$ spatial resolution over $26 \mathrm{~km}$ SMF using low-repetition-rate cyclic pulse coding” Opt. Lett., vol. 36, no. 13, pp. 2557-2559, 2011.

[2] J. Park et al., "Raman-based distributed temperature sensor with simplex coding and link optimization," Photon. Tech. Lett., vol. 18, no. 17, pp. 1871-1881, 2006.

[3] M. A. Soto et al., "Distributed temperature sensor system based on Raman scattering using correlation-codes," IEE Electron. Lett., vol. 43, no. 16 , pp. $862-864,2007$.

[4] A. Signorini et al., "40 km long-range Raman-based distributed temperature sensor with meter-scale spatial resolution," in Optical Fiber Communication Conference (OFC) 2010, San Diego, US, March 2010.

[5] M. A. Soto, G. Bolognini, F. Di Pasquale, and L. Thévenaz, "Simplexcoded BOTDA fiber sensor with $1 \mathrm{~m}$ spatial resolution over a $50 \mathrm{~km}$ range," Opt. Lett., vol. 35, no. 2, pp. 259-261, 2010.

[6] M. Webster, L. Raddatz, I. H. White, and D. G. Cunningham, "A Statistical Analysis of Conditioned Launch for Gigabit Ethernet Links Using Multimode Fiber," J. Lightwave Technol., vol 17, no. 9, pp. 1532-1541, 1999.

[7] P. C. Wait, K. De Souza, and T. P. Newson, "Theoretical comparison of spontaneous Raman and Brillouin based fibre optic distributed temperature sensors," Opt. Comm., vol. 144, pp.17-23, 1997. 